\title{
Cultivating cultural humility in social work teaching: What can be done differently?
}

\section{Saumya Tripathi ${ }^{1}$}

${ }^{1}$ Graduate School of Social Service, Fordham University

Received 29 June 2021

Accepted for publication 8 July 2021

Published 16 August 2021

\begin{abstract}
As an Indian social work instructor in the United States, I came across cultural differences between me and my students. This reflection paper aims to share culturally humble teaching practices that I have utilized, and how they aided me in overcoming and understanding cultural differences in the classroom. This reflection may help international instructors teaching in a multicultural context and who experience similar cultural differences in their academic journey.
\end{abstract}

Keywords: cultural humility, social work, instructor, teaching, multicultural context

\section{Background}

Throughout our lives we move between several cultures without acknowledging it; for me, it was the Indian culture that shaped my positionality as a social work instructor. The first cultural gap I encountered was in the summer of 2017 during one of my research classes. I was elaborating on a critical topic of choosing research design when I noticed that students were eating food in my class. In another class, a student asked me out for a coffee meeting outside the school to learn about my work and research experiences. Coming from an Indian cultural background, I was dumbstruck. It took me a few weeks to process this activity in my lectures; I started questioning myself: 'Is my content or teaching boring?', 'Am I too young to teach?', 'Am I able to maintain discipline and authority?'. Such questions bothered me for a long time because, in an Indian scenario, such gestures are not only considered inappropriate and unacceptable but a signal of disrespect towards others. My expectation of maintaining classroom discipline was once again challenged when a student addressed me by shortening my first name and argued for a better grade before the final exams. Later, several similar instances occurred, but I felt clueless of the experiences and how to address it.

In social work classrooms, acknowledging our positionality requires recognizing intersecting social locations based on race, class, gender, sexuality, ability status, and complex power dynamics between our students and us that influence and potentially bias our understanding of the world (Ambrose et al., 2010). As social work instructors, we heavily emphasize on cultural competence, a framework for advancing cultural sensitivity through awareness of cultural differences and their impact on an individual's values, beliefs, perspective (Danso, 2016). However, in my case, something more was needed that is moving beyond the idea of just understanding other's cultural differences and focusing on one's own positionality and its influence on own cultural differences. Cultural humility postulates that each individual in the teaching and learning environment brings a wealth of human experience, informed and shaped by language, traditions, and practices - or different ways of perceiving and understanding the world (Arvanitakis, 2014). It requires instructors to continually engage in self-reflection and selfcritique as lifelong learners.

\section{The journey of adapting culturally humble teaching methods}

To be culturally adaptive, I started taking students' evaluations quite early in the semester. I sometimes would even ask them directly if I can make the content clear. I would ask whether my lecturing style is boring, or if there is any other teaching method they prefer over lecturing and 
PowerPoint slides. Surprisingly, they all liked my teaching style and greatly appreciated the examples and real-life work experiences that I would bring to the classroom. Still, here I was, cautious in my teaching practice and unable to overcome the cultural differences. After a year, during class, I saw a student dropping the book to the floor and accidentally stepping over it. Later, when the session was finished, I picked the book up and touched it as a sign of respect in my Indian culture, which many students standing there found 'weird'. This particular event marked my pursuit to focus on my cultural values and positionality and be culturally humble.

Cultural humility postulates that everyone who grows up in a social environment carry some hidden biases (Gottlieb, 2020). Three factors that guides one's development toward cultural humility are: (1) lifelong commitment to selfevaluation, (2) fix power imbalances, and (3) develop partnerships with people (Tervalon and Murray-Garcia, 1998). Based on these factors, I mainly focused on the following strategies to enhance cultural humility: (1) reflexivity of the self, profession, and students, (2) mindfulness for greater insight, and (3) building bridges with the students.

\subsection{Reflexivity of the self, profession, and students}

As a woman of color with diverse teaching and practice experience, I realize my differences stemmed from cultural variations that assign different social interpretations to a word, action, or phenomenon. For example, to a courtesy question of "how are you?" an Indian instructor may feel obliged to provide a detailed response without recognizing that it was only a greeting question in an American context. I was continually exploring my own social location and understanding the interplay of my identity that shapes beliefs regarding what I and others consider 'normal' or 'appropriate'. I started with a deep reflection process that focused on my values, perspectives, and biases derived from my own cultural origin and my professional experience working with individuals and communities in India. During this process, I retrieved the memories of my schooling years, college, and working life and explored how those have shaped my views of others and life altogether. The process helped me tremendously in transitioning my understanding towards my identity and its relationship with my students' identity and cultural value alignment. I witnessed a positive change in my professional relationship with students. I was simply not attributing things to wrong reasons. The next time students asked me to meet outside of school on coffee, I was flexible enough to understand their professional endeavors such as preparation for internship or job, at the same time delineating the boundary between professional vs. personal.

\subsection{Mindfulness for greater insight}

On any regular day, I start on an autopilot mode, going from one task to another, with little or no recognition of my attitudes, assumptions, and judgments. Mindfulness is defined as 'the awareness that arises through intentionally attending in an open, caring, and non-judgmental way' (Shapiro and Carlson, 2009, p.4). Initially, the whole process appeared time-consuming However, simply taking out 10 minutes during my teaching preparation time helped me practice mindfulness. I was able to experience self-awareness by emphasizing ongoing thoughts and resulting emotions from them. I felt an increased sensitivity towards student's backgrounds and the general classroom academic environment. The process helped me eliminate biases, stereotypes, self-narratives, judgments, and mental filters. The constant struggle of cultural differences in the teaching system was transformed into a constructive dialogue phase, interpersonal attributes of empathy, recognizing my students' emotions and learning from each of them.

\subsection{Building bridges with the students}

As instructors, we emphasize on our inter- and intrapersonal skills so that our students can approach us freely and without hesitation. However, after experiencing the cultural differences mentioned above, I further reflected on whether I can comfortably speak to my students and if they are as easily approachable to me - if not, why? The different kinds of power that automatically come with the teaching position need investigation to bridge these cultural differences. Acknowledging that each student contributes something different and unique to the classroom learning helped in demystifying the myth of power inherent in my unconscious mind. I started identifying the uniqueness of each student, recognizing that each one of them has a life and story of their own that brings them to the social work class and reflected on how I can relate to them. In the past, whenever students asked me my personal phone number, I was reluctant to share it. I considered it unprofessional, but it's only after exploring the cultural dimension in a humble way I was able to check the power imbalances in the dynamics of our communication by using student-centered learning.

\section{Final comments}

Incorporating cultural humility into our teaching practices is a process of constant self-reflection and lifelong inquiry. Central to the process of practicing cultural humility is the instructor's own ability to ponder over their cultural values and associated biases purposefully. After practicing it for more than three years, I feel the reward of teaching in a multicultural 
classroom outweigh the initial challenges that I experienced due to cultural differences in the classroom.

\section{References}

Ambrose, S.A., Bridges, M.W., DiPietro, M., Lovett, M.C. and Norman, M.K. (2010) How learning works seven research-based principles for smart teaching. San Francisco, CA: John Wiley \& Sons.

Arvanitakis, J. (2014) On cultural humility. Available at: http://jamesarvanitakis.net/10coffeeshots/on-culturalhumility/ (Accessed: 9 May 2021 ).

Danso, R. (2016) 'Cultural competence and cultural humility: A critical reflection on key cultural diversity concepts', Journal of Social Work, 18(4), pp. 410-430.

Gottlieb, M. (2020) 'The case for a cultural humility framework in social work practice', Journal of Ethnic \& Cultural Diversity in Social Work. DOI: 10.1080/15313204.2020.1753615

Shapiro, S. and Carlson, L. (2009) The art and science of mindfulness. Washington, D.C: American Psychological Association.

Tervalon, M. and Murray-García, J. (1998) 'Cultural humility versus cultural competence: A critical distinction in defining physician training outcomes in multicultural education', Journal of the Health Care for the Poor and Underserved, 9(2), pp. 117-125. 